cients $\delta_{33}$ and $\delta_{15}=\delta_{31}$ for several ferroelectrics relative to a quartz standard $\left(\delta_{11}^{\boldsymbol{Q}}\right)$. To compare predicted absolute values with experiment we note that only $d_{36}$ of $\mathrm{NH}_{4} \mathrm{H}_{2} \mathrm{PO}_{4}$ (ADP) has been measured absolutely with any degree of precision. We use this value $^{12}\left(d_{36}=5.0 \pm 0.6 \times 10^{-24} \mathrm{mks}\right)$ together with Miller's tabley to obtain $\delta_{11}^{Q} \approx 0.25 \pm 0.05 \times 10^{10} \mathrm{mks}$, from which we can convert the relative values in Table I to absolute values. We tabulate in the last four columns of Table I these experimental values and also the theoretical results obtained by substituting the $g$ coefficients given above into Eq. (12).

The calculated results shown in Table $I$ are generally somewhat higher than the experimental results suggesting that $\beta$ at optical frequencies $\left(\beta^{o}\right)$ is somewhat smaller than the static value $\left(\beta^{s}\right)$. Examination of the experimental anisotropy ratio $\delta_{33} / \delta_{15}$ shows that $\delta_{33} / \delta_{15} \approx 2$ in $C_{4 v}$ crystals (other than $\mathrm{BaTiO}_{3}$ (ref. 13)) whereas in $C_{6 r} \mathrm{LiNbO}_{3}$ and $\mathrm{LiTaO}_{3}$ the ratio is $\delta_{33}^{\prime} / \delta_{15}^{\prime} \sim 10$. Better agreement with experiment, in both absolute value and anisotropy, can be achieved by adjusting $\beta^{o}$ with respect to $\beta^{s}$, i.e., by letting $\beta_{11}^{o}=a \beta_{11}^{s}, \beta_{12}^{o}=b \beta_{12}^{s}$, and $\beta_{44}^{o}=$ $c \beta_{44}^{s}$. We then find by combining the experimental $\delta$ coefficients listed in Table I with Eqs. (2) and (12) that $a \approx 1 / 2$ and $b \approx 1$ for $C_{4 v}$ crystals; using these values for $C_{6 r}$ crystals we find $c \approx 1$. We conclude that $\beta_{11}^{o} \approx \frac{1}{2} \beta_{11}^{s} \approx 3 / \zeta^{3}, \beta_{12}^{o} \approx \beta_{12}^{s} \approx 1.5 / \zeta^{3}$, and $\beta_{44}^{o} \approx$ $\beta_{44}^{s} \approx 4.4 / \zeta^{3} \mathrm{eV}-\mathrm{m}^{4} / \mathrm{C}^{2}$. These results show that static and electronic polarizations, as assumed initially, produce similar Stark shifts in the energy bands.

We thank S. Singh for kindly providing the data used in Table I. We also express our sincere thanks to $\mathrm{D}$. A. Kleinman for reading the manuscript and for helpful comments.

\footnotetext{
${ }^{1}$ N. Bloembergen, Nonlinear Optics (W. A. Benjamin, New York, 1965); C. G. B. Garrett and F. N. H. Robinson, J. Quantum Elect. QE-2, 328 (1966); S. K. Kurtz and F. N. H. Robinson, Appl. Phys. Letters 10, 62 (1967).

${ }^{2}$ F. N. H. Robinson, Bell System Tech. J. 46, 913 (1967).

${ }^{3}$ A. H. Kahn and A. J. Leyendecker, Phys. Rev. 135, A1321 (1964).

${ }^{4}$ J. R. Brews, Phys. Rev. Letters 18, 662 (1967).

${ }^{5}$ J. D. Zook and T. N. Casselman, Phys. Rev. Letters 17, 960 (1966).

${ }^{6}$ A. Frova and P. J. Boddy, Phys. Rev. 153, 606 (1967).

${ }^{7} \mathrm{M}$. DiDomenico, Jr. and S. H. Wemple (to be published).

${ }^{8}$ S. H. Wemple, M. DiDomenico, Jr., and I. Camlibel, Appl. Phys. Letters 12, 209 (1968).

${ }^{9}$ R. C. Miller, Appl. Phys. Letters 5, 17 (1964).

${ }^{10}$ D. A. Kleinman, Phys. Rev. 126, 1977 (1962).

"J. D. Zook, D. Chen, and G. N. Otto, Appl. Phys. Letters 11, 159 (1967).

${ }^{12}$ G. E. Francois, Phys. Rev. 143, 597 (1966); J. E. Bjorkholm and A. E. Siegman, Phys. Rev. 154, 51 (1967).

${ }^{13}$ The results for $\mathrm{BaTiO}_{3}$ agree approximately with our predictions; however, experimentally $\delta_{33}$ is about a factor of 4 too low for complete agreement with our model. There is evidence that this discrepancy may be related to the presence of an antiparallel domain structure in "poled" $\mathrm{BaTiO}_{3}$.
}

\title{
THE GAUSSIAN MODE IN OPTICAL RESONATORS WITH A RADIAL GAIN PROFILE*
}

\author{
Lee Caspersont and Amnon Yariv \\ Division of Engineering and Applied Science \\ California Institute of Technology \\ Pasadena, California 91109 \\ (Received 28 February 1968)
}

The dependence of the parameters of the Gaussian mode in laser resonators on the properties of the medium in the cavity is studied. Experimental verification of the theoretical results is presented. It is found that the modes in a high-gain laser may differ widely from the usual free space resonator results. Also, resonator configurations which in free space are unstable may, with a suitable medium, support low-loss Gaussian modes.

A theoretical study by Kogelnik ${ }^{1}$ and a related experiment of Klüver and Kogelnik² show that a

\footnotetext{
* Research supported by the Office of Naval Research through Contract Nonr 220(50).

$\uparrow$ National Aeronautics and Space Administration Predoctoral Trainee.
}

medium with a quadratic gain profile can support Gaussian optical beams. Confined beams are possible which propagate without spreading, i.e., the beam radius and the radius of curvature of the phase fronts are constant.

In the following we report a theoretical and an experimental study of the problem of the optical 
modes that can exist inside a resonator filled with a medium possessing a quadratic gain profile.

Consider a plane-parallel resonator of length $d$. The complex propagation constant of the medium is taken as

$$
k=k_{0}-\frac{1}{2} k_{2} r^{2}
$$

where $r$ is the radial distance from the axis of cylindrical symmetry $(z)$. Let $R(z)$ and $\omega(z)$ represent, respectively, the radius of curvature and beam radius of the Gaussian beam. ${ }^{3}$ We can define a complex beam parameter $q$ by

$$
\frac{1}{q(z)}=\frac{1}{R(z)}-i \frac{\lambda}{\pi \omega^{2}(z)}
$$

where $\lambda$ is the free space wavelength.

Kogelnik ${ }^{4}$ has shown that the parameter $q$ of a Gaussian beam propagating through a sequence of lens-like media evolves according to

$$
q_{2}=\frac{A_{T} q_{1}+B_{T}}{C_{T} q_{1}+D_{T}}
$$

where $q_{1}$ and $q_{2}$ are, respectively, the "input" and "output" beam parameters and where the coefficients are the elements of the paraxial ray matrix. The coefficients are found by forming the ordered product of the individual matrices which characterize the lens-like elements between the input and output.

The matrix corresponding to one round trip through the resonator described above is

$$
\left(\begin{array}{ll}
A_{T} & B_{T} \\
C_{T} & D_{T}
\end{array}\right)=\left(\begin{array}{ll}
A & B \\
C & D
\end{array}\right)\left(\begin{array}{ll}
1 & 0 \\
0 & 1
\end{array}\right)\left(\begin{array}{ll}
A & B \\
C & D
\end{array}\right)\left(\begin{array}{ll}
1 & 0 \\
0 & 1
\end{array}\right)
$$

where the first and third matrices on the right side of (4) correspond to one passage through the medium, while the second and fourth matrices represent reflections at the plane parallel end reflectors.

For a medium of length $d$ described by (1) the $(A B C D)$ matrix of $(4)$ can be written $a^{5}$

$$
\left(\begin{array}{ll}
A & B \\
C & D
\end{array}\right)=\left[\begin{array}{cc}
\cos \left(\sqrt{\frac{k_{2}}{k_{0}} d}\right) & \sqrt{\frac{k_{0}}{k_{2}} \sin }\left(\sqrt{\frac{k_{2}}{k_{0}} d}\right) \\
-\sqrt{\frac{k_{2}}{k_{0}} \sin \left(\sqrt{\frac{k_{2}}{k_{0}} d}\right)} & \cos \left(\sqrt{\frac{k_{2}}{k_{0}} d}\right)
\end{array}\right]
$$

so that the matrix product (4) is the same as (5) except that $d$ is replaced by $2 d$.

To obtain the steady-state beam parameter we set $q_{2}=q_{1}=q$ in (3) which, using (4) and (5), leads to

$$
\frac{1}{q}=\mp i \sqrt{\frac{k_{2}}{k_{0}}} \text {. }
$$

If the radial variation of $k$ is due to the gain only, we may rewrite (1) as

$$
\begin{aligned}
& k_{0}=\beta_{0}+i \alpha_{0}, k_{2}=i \alpha_{2}, \sqrt{\frac{k_{2}}{k_{0}}}=\frac{(1+i)}{2} \sqrt{\frac{\alpha_{2} \lambda}{\pi}} \\
& \text { for } \beta_{0}=\frac{2 \pi}{\lambda} \gg \alpha_{0} .
\end{aligned}
$$

Substituting in (6) and using (2) yields

$$
\frac{\pi \omega^{2}}{\lambda}=2 \sqrt{\frac{\pi}{\alpha_{2} \lambda}}
$$

for the (squared) beam radius at the mirror. This result is independent of the location of the reference plane, so that the beam radius $\omega$ is a constant throughout the length of the resonator. Thus a resonator which in free space has high diffraction losses can support a stable low-loss Gaussian mode when filled with a medium having a quadratic gain profile.

The experimental setup used to test the theory is shown in Fig. 1. The high-gain xenon laser oscillating at $3.5 \mu$ provides a gain constant whose radial dependence is described by the zero-order Bessel function. ${ }^{6}$ Near the axis we may use the quadratic expansion to obtain

$$
\alpha_{2}=\alpha_{0} \frac{2.88}{r_{0}^{2}}
$$

where $\alpha_{0}$ is the gain constant on the axis $(r=0)$ and $r_{0}$ is the radius of the discharge tube. In our experiment $r_{0}=2.75 \mathrm{~mm}$.

Curve (b) of Fig. 2 is a plot of Eq. (7). The experimental points shown were determined by radial scanning, with a small aperture, of the output beam with the laser operating just above threshold to avoid appreciable gain saturation. The gain on axis $\left(\alpha_{0}\right)$ was determined from the known insertion loss and includes a self-consistent correction for the gain profile. The good agreement of the data with the theoretical curve constitutes a verification of Kogelnik's propagation theory. ${ }^{1}$

Curves $(a)$ and $(c)$ are theoretical plots of the beam radius at the left mirror of the "unstable" and "stable" resonators shown in Fig. 2. The curves are obtained by a procedure analogous to that leading to Eq. (7) except that the second $\left(\begin{array}{ll}1 & 0 \\ 0 & 1\end{array}\right)$ matrix on

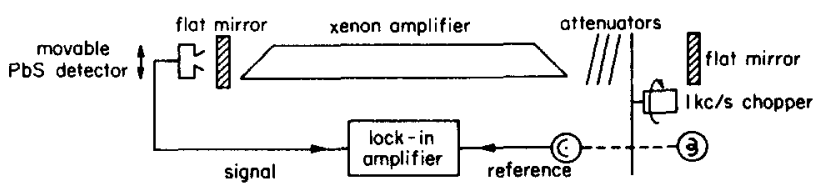

Fig. 1. Experimental setup. 
the right side of (4) is replaced by $\left(\begin{array}{cc}1 & 0 \\ -2 / R & 1\end{array}\right)$ to account for the curvature of the left reflector. Note that for large gains curves $(a),(b)$, and $(c)$ are identical so that for the high-gain case the beam radius becomes independent of the mirrors' curvatures. The otherwise unstable resonator $(a)$ has been rendered stable by the quadratic gain medium. In the low-gain limit, the curve corresponding to an

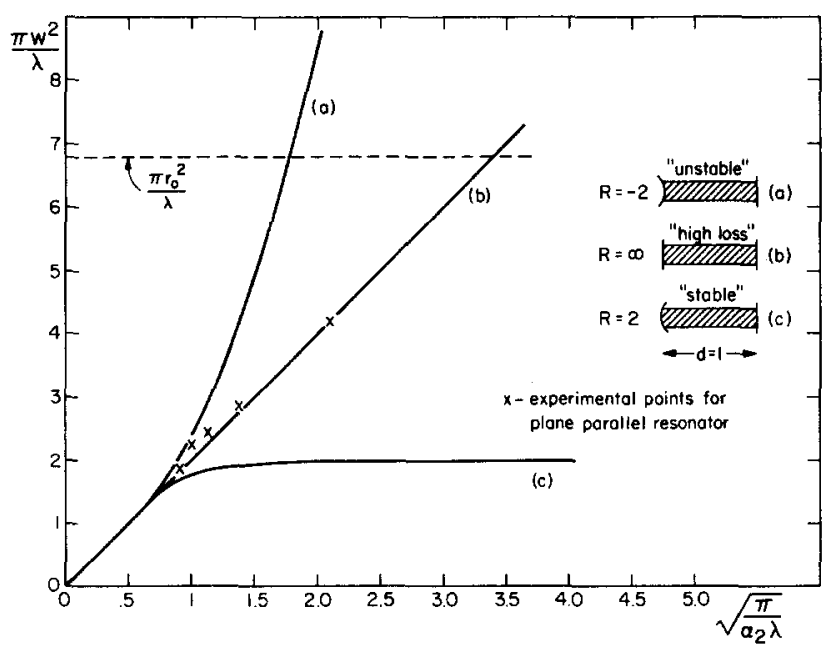

Fig. 2. Theoretical curves showing the dependence of the beam radius at the left mirror on the gain constant $\alpha_{2}$. Experimental points for the plane-parallel resonator are also shown. unstable resonator increases quadratically, while the curve corresponding to a stable configuration approaches the usual empty resonator value as should be expected.

In summary, the theory of Gaussian beam propagation in media with an index profile ${ }^{1}$ is used to derive the mode characteristics of high-gain laser resonators. We find theoretically and experimentally that for sufficiently high quadratic gain $\alpha_{2}$ the beam radius (and radius of curvature) are essentially independent of the mirrors' curvatures and separation. Low (diffraction) loss modes thus exist in configurations that may otherwise be unstable. Moreover, the agreement of the data with the theory provides a verification of the latter.

The authors acknowledge the excellent technical support of D. Armstrong and thank Dr. P. Clark for supplying the laser used in the experiment.

\footnotetext{
${ }^{1}$ H. Kogelnik, Appl. Opt. 4, 1562 (1965).

${ }^{2} \mathrm{~W}$. Klüver and H. Kogelnik, paper presented at Conference on Electron Device Research, Pasadena, June 1966 (unpublished).

${ }^{3}$ G. Goubau and F. Schwering, Inst. Radio Engrs. Trans. AP-9, 248 (1961) or A. Yariv, Quantum Electronics (John Wiley and Sons, Inc., 1967), p. 224.

${ }^{4}$ H. Kogelnik, Bell System Tech. J. 44, 455 (1965).

${ }^{5}$ Equation (5) is obtained from Eq. (34) of ref. 1 by the substitution $q=k_{0} / Q$ and use of our Eq. (3).

${ }^{6}$ W. R. Bennett, Appl. Opt. Suppl. 2, Chemical Lasers, 3 (1965).
}

\section{A WIDE-RANGE VARIABLE-FREQUENCY GUNN OSCILLATOR}

William H. Haydl

Science Center/Aerospace and Systems Group North American Rockwell Corp. Thousand Oaks, California 91360 (Received 15 April 1968)

By controlling the illumination intensity along a sample of photoconducting GaAs, the point of nucleation of the high field domain has been varied. When a very thin line shadow was projected on an illuminated sample, the oscillation frequency corresponded to the distance between the shadow and the anode and could be varied continuously over a range of about 5:1 by varying the position of the shadow along the sample.

Variable frequency oscillators which are based on the properties and the propagation characteristics of the high electric field domain in GaAs have been reported by several authors. ${ }^{1-3}$ The frequency of such devices in which a traveling high electric field domain causes periodic fluctuations in the current may be controlled by varying the domain velocity or by varying the distance that the domain travels. The small variation of the frequency with sample voltage of Gunn oscillators when in a resistive circuit is well known and is believed to be caused by the variation of the domain velocity. ${ }^{4}$ Larger frequency variations are possible by varying the point of nucleation or the point of collapse of the domain. Tuning ranges of about 2:1 have been obtained by using tapered diodes which exhibit a decreasing electric field from cathode to anode. ${ }^{3}$ The taper may be achieved by a varying geometry or a varying 